\title{
Effects of Different Systolic Blood Pressure Targets on Myocardial Function: A One-Year Follow-Up in Geriatric Hypertension
}

\author{
Xiaoyan Chen' \\ Qingmei Yang' \\ Jianxiu Fang \\ Haifeng Guo ${ }^{2}$ \\ 'Department of Ultrasound, Shanxi \\ Bethune Hospital \& Shanxi Academy of \\ Medical Sciences, Taiyuan, Shanxi \\ Province, People's Republic of China; \\ ${ }^{2}$ Department of Medical Imaging, Shanxi \\ Armed Police Force Hospital, Taiyuan, \\ Shanxi Province, People's Republic of \\ China
}

Background: A lower systolic blood pressure (SBP) target reduces major cardiovascular events and mortality from any cause of geriatric hypertension. However, the effect of different SBP targets on myocardial function remains unclear. This study aimed to determine changes in left ventricular (LV) strain in older hypertensive patients after 1 year of different SBP goals, and to evaluate its effects on myocardial mechanics in this population.

Methods: We studied 313 hypertensive adults aged 60 years or older after 1 year of the Systolic Blood Pressure Intervention Trial. They were divided into the intensive group (target SBP: $110-130 \mathrm{mmHg}$ ) and the standard group (target SBP: $130-150 \mathrm{mmHg}$ ). All participants underwent echocardiography within 1 week after enrollment and 1 year after participating in the study. Global longitudinal strain (GLS) of the LV (endocardial, middle, and epicardial layer: GLS-end, GLS-mid, and GLS-epi, respectively) and the improvement of GLS at 1 year ( $\Delta$ GLS-end, $\Delta$ GLS-mid, and $\Delta$ GLS-epi) were measured.

Results: At 1 year, GLS-end in the intensive group was slightly improved compared with that before the trial $(-23.78 \% \pm 3.10 \%$ vs $-22.58 \% \pm 3.11 \%, \mathrm{P}<0.05)$. The $\Delta$ GLS-end and $\Delta$ GLS-mid in the intensive group were higher than those in the standard group (1.20 $\pm 0.23 \mathrm{vs}$ $0.58 \pm 0.59 \%$ and $0.70 \pm 0.21$ vs $0.52 \pm 0.17, \mathrm{P}<0.05$ ). Moreover, SBP at 1 year and an angiotensin II type 1 receptor antagonist were independent factors that affected $\Delta$ GLS-end ( $\beta=$ $-0.005, \mathrm{P}=0.004 ; \beta=0.080, \mathrm{P}<0.001$, respectively).

Conclusion: These trial results suggest that a lower SBP target can slightly improve myocardial function in older hypertensive patients at 1 year.

Keywords: hypertension, blood pressure target, elder population, strain, left ventricular function

\section{Introduction}

Hypertension affects more than 1.2 billion individuals worldwide and has become the most critical public health problem. ${ }^{1}$ This condition is a major risk factor for cardiovascular events worldwide, especially in older patients with hypertension. ${ }^{2}$ Treating high blood pressure can significantly reduce the risk of cardiovascular disease, including stroke, myocardial infarction, and heart failure. ${ }^{3,4}$ However, at present, there is no unified international blood pressure control target for older hypertensive patients. The European Society of Hypertension and the European Society of Cardiology target a systolic blood pressure (SBP) of $<140-150 \mathrm{mmHg}$ with lower goals in fit and healthy patients. ${ }^{5}$ The 2017 American College of Cardiology/American Heart Association guidelines for hypertension suggest that
Correspondence: Xiaoyan Chen Department of Ultrasound, Shanxi Bethune Hospital \& Shanxi Academy of Medical Sciences, Taiyuan, Shanxi Province, People's Republic of China Tel +8613934200119

Email xiaoguoguol19@163.com 
for a general healthy age $\geq 65$ years, the blood pressure control target should be $<130$ mmHg. ${ }^{6}$ The 2019 Chinese Hypertension Guidelines consider that the blood pressure of older patients should be reduced to $<150 / 90 \mathrm{mmHg}{ }^{7}$

Guidelines for hypertension vary and which blood pressure target is better for heart function remains unknown. Findings from the Systolic Blood Pressure Intervention Trial (SPRINT) showed that intensive lowering of blood pressure (SBP $<120 \mathrm{mmHg}$ ) was associated with lower rates of cardiovascular events and mortality in hypertensive fit and frail older subjects compared with standard treatment. ${ }^{8}$ These findings suggested that lowering of blood pressure was beneficial for cardiac function. ${ }^{9}$ However, there is a high incidence of adverse events, such as hypotension, syncope, electrolyte disturbance, and acute renal failure, in the short term of intensive hypotension, and these can impair cardiac function. ${ }^{10,11}$ It has even been argued that antihypertensive treatment leads to a disproportionate reduction of contractile elements, leaving a relatively large number of noncontractile elements, which may lead to cardiac dysfunction. $^{12,13}$ Simultaneously, recent findings by D'Amato et al showed that intensive blood pressure reduction has no effect on target organ damage. ${ }^{14}$

Whether a lower or higher SBP target is associated with better LV myocardial function in older adults is currently unknown. Myocardial strain obtained using speckle-tracking echocardiography can quantify left ventricular (LV) function. ${ }^{15}$ The best evaluated strain parameter is longitudinal strain (LS), which is more sensitive than the LV ejection fraction in adult hypertension. ${ }^{15,16}$ In this study, we used speckle-tracking echocardiography to evaluate myocardial LS in older hypertensive patients who were enrolled in the SPRINT for 1 year. We aimed to assess the effects of different antihypertensive targets on LV cardiac function in geriatric hypertension, with an emphasis on myocardial mechanics.

\section{Methods}

\section{Study Participants}

In this prospective study, we recruited older hypertensive patients who were treated in our hospital from July 2019 to October 2019. Inclusion criteria were as follows: (1) patients with primary hypertension, including newly diagnosed hypertension, with an average follow-up (3 times) of outpatient SBP $\geq 140 \mathrm{mmHg}$, and patients undergoing antihypertensive treatment; (2) patients of Han ethnicity, aged 60-80 years; and (3) patients signed an informed consent form. Exclusion criteria were as follows: (1) SBP $\geq 190 \mathrm{mmHg}$ or diastolic blood pressure $<60$ mmHg; (2) confirmed secondary hypertension; (3) a history of stroke or acute myocardial infarction in the past 6 months, those who had revascularization surgery performed or planned within the next 6 months, or those with persistent atrial fibrillation or arrhythmia that affected blood pressure measurement with heart failure; (4) severe valve disease, cardiomyopathy, rheumatic heart disease, or congenital heart disease; (5) poor diabetes control, severe liver and kidney disease, or a history of malignant tumors; (6) patients with cognitive dysfunction or inability to take care of themselves; (7) participation in other clinical trials; (8) poor image quality that affected analysis; and (9) participants without complete data. Informed consent was provided by the patients and the study protocol was approved by the medical ethics committee.

Using blood pressure control goals, the patients were divided into the intensive group (target SBP: 110-130 $\mathrm{mmHg}$ ) and the standard group (target SBP: 130-150 $\mathrm{mmHg}$ ) if achievable without undue burden. All participants downloaded the mobile phone blood pressure management software "Hypertension Doctor", reported blood pressure daily, and managed by a cardiologist. Under the condition that the patient could tolerate the procedure, blood pressure was gradually adjusted within 3 months to reach the corresponding target in each group. We recorded the systolic and diastolic blood pressures of the subjects on the three mornings before and one year after participating in the trial, and obtained their average values as the systolic and diastolic blood pressures for the two time periods.

In total, the study included 342 older hypertensive patients (age, 60-80 years), including 173 in the intensive group and 169 in the standard group. After 1 year of follow-up, there were 159 patients in the intensive group and 154 patients in the standard group. Reasons for loss to follow-up were as follows: 3 patients had acute cardiovascular events, 2 had breast cancer requiring chemotherapy, 6 had arrhythmia, and 17 could not be contacted or voluntarily left the study.

\section{Laboratory Analysis}

Biochemical analyses, including measurement of total cholesterol, triglyceride, low-density lipoprotein, and highdensity lipoprotein levels, were performed in all patients. 


\section{Echocardiography}

Echocardiographic imaging was performed using the Vivid E9 GE Medical Systems commercial scanner (GE Vingmed Ultrasound AS, Norway), which was equipped with a $5 \mathrm{~S}$ probe (1-5 MHz). All patients underwent echocardiography within 1 week after enrollment and after 12 months of participation in the study, according to the recommendations of the American Society of Echocardiography. ${ }^{17}$ LV enddiastolic diameter (LVD), LV end-systolic diameter (LVDs), and end-diastolic inter-ventricular septal (IVSd) and LV posterior wall thickness (PWTd) were measured with on the parasternal long axis. The LV mass (LVM) was calculated according to a previously published methodology, ${ }^{17} \mathrm{LVM}(\mathrm{g})$ $=0.8 \times 1.04\left[(\mathrm{LVDd}+\mathrm{IVSd}+\mathrm{PWTd})^{3}-\mathrm{LVDd}^{3}\right]+0.6$. The LV mass index was calculated as follows: $\mathrm{LV}$ mass index $=\mathrm{LVM} /$ body surface area. ${ }^{17}$ Early and late mitral valvular blood flow velocity peaks ( $\mathrm{E}$ and $\mathrm{A}$, respectively) were measured by pulsed-wave Doppler, and mitral annular early and late peak velocities (Em and Am, respectively) were measured by tissue Doppler. The E/A and E/Em were then calculated. The LV ejection fraction was measured by the Simpson biplane method.

After acquiring the apical long axis and four- and twochamber views of three consecutive cardiac cycles (frame rate 60-120), the different views were analyzed using Echo PAC analysis software (version: 201). We sketched the subendocardial area of each view. The software was used to automatically create a region of interest, which contained subendocardial, middle, and subepicardial areas, and we adjusted the region of interest to include the complete LV myocardium. The software performed speckle tracking analysis on the LV myocardium in each view. Upon delineating the region of interest, the software automatically generated time-domain strain curves in six segments with which endsystolic strain was subsequently calculated. Global longitudinal strain (GLS) was defined as the average longitudinal strain at the end-systole in 18 segments.

Image analysis was performed by two experienced echocardiogram technicians, who were not aware of the grouping of subjects. After this analysis, we obtained GLS of the LV endocardial layer, middle layer, and epicardial layer (GLS-end, GLS-mid, and GLS-epi, respectively) (Figure 1). Figure 2 shows that we calculate the improvement of the strain value after 1 year of treatment in all patients ( $\Delta$ GLS: $\Delta$ GLS-end, $\Delta$ GLS-mid, and $\Delta$ GLS-epi, respectively): $\Delta \mathrm{GLS}=\mid$ GLS $(1$ year after joining this trial) - GLS (before joining the trial)|.

\section{Statistical Analysis}

All statistical analyses were performed with SPSS 17.0 (SPSS Inc., Chicago, IL). Continuous data are presented as mean \pm SD. Frequencies are expressed as percentages. The Student's $t$-test was used as appropriate for comparison of continuous data between the two groups. The chi-square test was used to compare the variables of sex and medication between the two groups. The paired $t$-test was used as appropriate for comparison of continuous data before and after participating in this trial. Pearson's correlation was chosen to test correlations among the clinical dates, LV structure, LV function parameters, and strain parameters. Independent determinants of LV myocardial strain parameters were examined using multivariate stepwise linear regression. P values $<0.05$ were considered to be statistically significant.

For reliability of the GLS-end, GLS-mid, and GLS-epi results, the intra-class correlation coefficient (ICC) was used to evaluate inter- and intra-observer variability. Twenty patients were randomly chosen for this analysis. Clinical significance was categorized as follows: good, ICC $\geq 0.75$; moderate, ICC $\geq 0.4$ and $<0.75$; and poor, ICC $<0.4$.

\section{Results}

\section{Baseline Characteristics of the Two Groups of Participants}

The clinical characteristics of the two groups of participants are shown in Table 1. No significant difference was found in sex distribution, age, body mass index, body surface area, blood pressure, heart rate, duration of hypertension, proportion of diabetes, and glucose, triglyceride, cholesterol, high-density lipoprotein, or low-density lipoprotein levels between the intensive and standard groups.

General parameters of echocardiography between the two groups are shown in Table 2. There were no significant differences regarding structural and conventional functional parameters of the LV between the two groups. The left ventricular ejection fraction and E/Em were in the normal range in the groups.

There were also no significant differences in GLS-end, GLS-mid, and GLS-epi between the two groups (Table 3).

\section{Comparison of Antihypertensive Medication Between the Two Groups of Patients After Enrolling in the Trial}

To achieve the target blood pressure of each group, we adjusted the medication regimen according to the patient's 

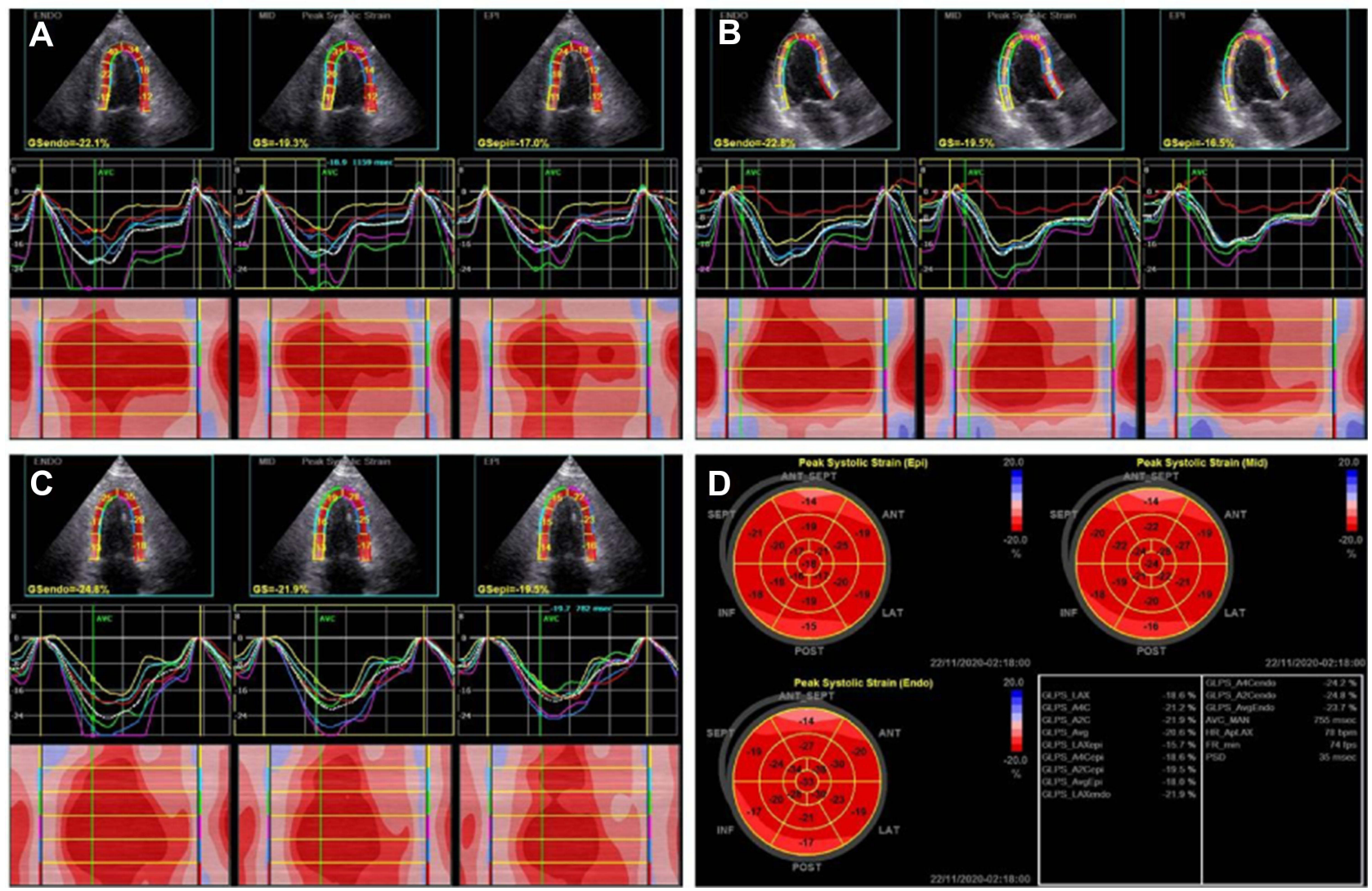

Figure I Strain analysis image of a subject, (A) Left ventricular longitudinal strain curve of apical four-chamber view, (B) Left ventricular longitudinal strain curve of apical long axis view, (C) Left ventricular longitudinal strain curve of apical two-chamber view, (D) Bull's eye diagram of longitudinal strain of left ventricular endocardium, middle and epicardial layers.

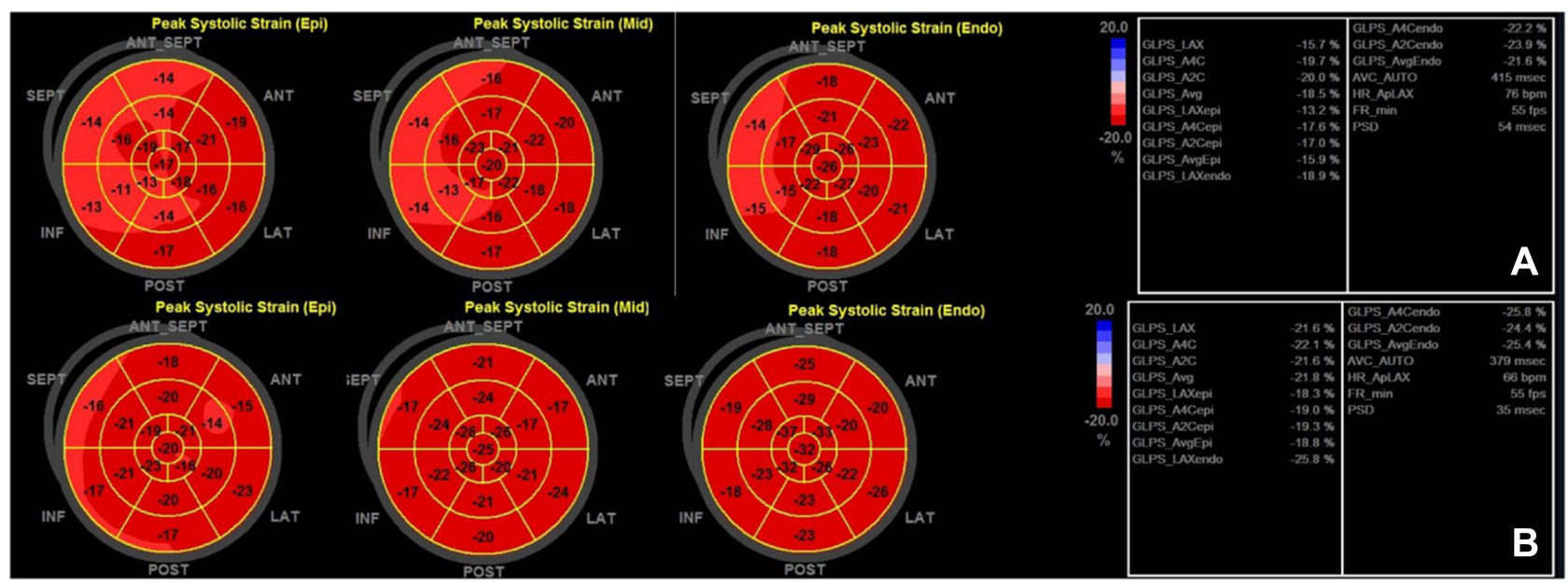

Figure 2 Left ventricular strain images of a patient before and after one year, (A) Before participating in this trial, (B) After participating in this trial for one year. Calculation improvement of the strain value after I year of treatment: $\Delta \mathrm{GLS}$-end=|-25.4- $-21.6 \mid=3.8(\%)$, GLS-mid=|-21.8- $-|8.5|=3.3(\%), \Delta \mathrm{GLS}-\mathrm{epi}=|-18.8--| 5.9 \mid=2.7(\%)$.

Abbreviations: GLS, global longitudinal strain; GLS-end, GLS-mid and GLS-epi, global longitudinal strain of left ventricular endocardial layer, middle layer and epicardial layer.

individual situation. Comparison of the distribution of an angiotensin II type 1 receptor antagonist (olmesartan medoxomil tablets), calcium antagonist (amlodipine besylate tablets), and diuretic (hydrochlorothiazide) in the two groups is shown in Table 4. The rate and dosage of angiotensin II type 1 receptor antagonists and diuretics in 
Table I Before Participating in This Trial Characteristics of the Participants

\begin{tabular}{|c|c|c|c|c|}
\hline Parameter & Intensive Group $n=159$ & Standard Group $n=154$ & $\mathbf{t} / \chi^{2}$ & $P$ value \\
\hline Age (years) & $66.23 \pm 4.98$ & $66.23 \pm 4.76$ & -0.002 & 0.999 \\
\hline Male gender, n (\%) & $69(43.4 \%)$ & $72(46.8)$ & 0.356 & 0.571 \\
\hline High $(\mathrm{cm})$ & $162.89 \pm 7.28$ & $163.23 \pm 7.78$ & -0.404 & 0.687 \\
\hline Weight (Kg) & $67.82 \pm 9.89$ & $69.78 \pm \mid 1.51$ & $-1.6 \mid 4$ & 0.108 \\
\hline Body mass index $\left(\mathrm{Kg} / \mathrm{m}^{2}\right)$ & $25.54 \pm 3.09$ & $26.10 \pm 3.31$ & -1.553 & 0.121 \\
\hline Body surface area $\left(\mathrm{m}^{2}\right)$ & $1.73 \pm 0.15$ & $1.73 \pm 0.18$ & -0.223 & 0.824 \\
\hline Waistline(cm) & $88.73 \pm 9.75$ & $90.64 \pm 9.74$ & -1.739 & 0.083 \\
\hline Heart rate (beats/min) & $74.43 \pm 11.43$ & $73.73 \pm 12.49$ & 0.518 & 0.605 \\
\hline $\mathrm{SBP}(\mathrm{mm} \mathrm{Hg})$ & $143.70 \pm 20.65$ & $143.43 \pm 14.42$ & 0.134 & 0.893 \\
\hline $\mathrm{DBP}(\mathrm{mm} \mathrm{Hg})$ & $80.83 \pm 9.92$ & $82.43 \pm 9.65$ & -1.445 & 0.150 \\
\hline $\mathrm{SBP}-\max (\mathrm{mm} \mathrm{Hg})$ & $|67.94 \pm| 3.9 \mid$ & $165.63 \pm 12.03$ & 1.568 & 0.118 \\
\hline DBP - max (mm Hg) & $99.46 \pm 11.65$ & $99.91 \pm|2.4|$ & -0.334 & 0.739 \\
\hline Plasma triglycerides $(\mathrm{mmol} / \mathrm{l})$ & $2.01 \pm 1.14$ & $1.86 \pm 1.06$ & 1.203 & 0.230 \\
\hline Total cholesterol (mmol/l) & $4.50 \pm 0.90$ & $4.44 \pm 1.01$ & 0.585 & 0.559 \\
\hline Low-density lipoprotein (mmol/l) & $1.63 \pm 1.10$ & $1.52 \pm 0.82$ & 1.021 & 0.308 \\
\hline High-density lipoprotein ( $\mathrm{mmol} / \mathrm{l})$ & $2.68 \pm 0.75$ & $2.61 \pm 0.83$ & 0.767 & 0.444 \\
\hline Hypertension duration (years) & $16.95 \pm 8.86$ & $15.55 \pm 7.64$ & 1.503 & 0.134 \\
\hline
\end{tabular}

Note: Data presented as mean \pm standard deviation or $\mathrm{n}(\%)$.

Abbreviations: SBP, systolic blood pressure; DBP, diastolic blood pressure.

Table 2 Left Ventricular Structure and Function Before and After Joining This Trial of the Two Groups

\begin{tabular}{|c|c|c|c|c|}
\hline Parameter & Intensive Group n= I59 & Standard Group $n=154$ & $\boldsymbol{t}$ & $P$ value \\
\hline \multicolumn{5}{|c|}{ Before participating in this trial } \\
\hline IVSd(mm) & $10.16 \pm 1.83$ & $10.00 \pm 1.66$ & 0.786 & 0.433 \\
\hline PWTd(mm) & $10.11 \pm 1.92$ & $10.04 \pm 2.03$ & 0.325 & 0.746 \\
\hline $\operatorname{LVDd}(\mathrm{mm})$ & $47.14 \pm 2.9$ & $47.54 \pm 3.13$ & -1.173 & 0.242 \\
\hline LVDs(mm) & $31.11 \pm 2.89$ & $30.9 \pm 3.04$ & 0.618 & 0.537 \\
\hline LVEF (\%) & $63.68 \pm 7.40$ & $64.97 \pm 6.69$ & -1.616 & 0.107 \\
\hline LVMI(g/m2) & $91.68 \pm 9.6$ & $92.28 \pm 9.76$ & -0.540 & 0.589 \\
\hline $\mathrm{E}(\mathrm{m} / \mathrm{s})$ & $58.63 \pm 4.78$ & $59.23 \pm 5.51$ & -1.039 & 0.300 \\
\hline $\mathrm{A}(\mathrm{m} / \mathrm{s})$ & $71.24 \pm 12.14$ & $73.44 \pm 14.10$ & -1.479 & 0.140 \\
\hline $\mathrm{Em}(\mathrm{m} / \mathrm{s})$ & $7.17 \pm 1.66$ & $7.20 \pm 2.07$ & -0.135 & 0.893 \\
\hline E/A & $0.86 \pm 0.21$ & $0.84 \pm 0.21$ & 0.472 & 0.637 \\
\hline $\mathrm{E} / \mathrm{Em}$ & $8.52 \pm 1.67$ & $8.70 \pm 1.89$ & -0.889 & 0.375 \\
\hline \multicolumn{5}{|c|}{$\begin{array}{l}\text { After participating in this trial for } \\
\text { one year }\end{array}$} \\
\hline $\operatorname{IVSd}(\mathrm{mm})$ & $9.64 \pm 1.84$ & $9.95 \pm 1.79$ & -1.554 & 0.121 \\
\hline PWTd(mm) & $9.57 \pm 1.75$ & $9.89 \pm 1.70$ & -1.632 & 0.104 \\
\hline $\operatorname{LVDd}(\mathrm{mm})$ & $47.04 \pm 4.96$ & $46.87 \pm 4.79$ & 0.308 & 0.758 \\
\hline LVDs(mm) & $30.17 \pm 3.03 *$ & $29.79 \pm 3.38^{*}$ & 1.060 & 0.290 \\
\hline LVEF(\%) & $66.77 \pm 7.5^{*}$ & $66.81 \pm 7.52 *$ & -0.046 & 0.963 \\
\hline LVMI(g/m2) & $91.44 \pm 8.07$ & $92.79 \pm 8.59$ & -1.427 & 0.155 \\
\hline$E(m / s)$ & $58.90 \pm 4.02$ & $59.24 \pm 4.26$ & -0.727 & 0.468 \\
\hline $\mathrm{A}(\mathrm{m} / \mathrm{s})$ & $70.78 \pm 9.77$ & $67.80 \pm 8.83 *$ & 2.827 & 0.005 \\
\hline $\mathrm{Em}(\mathrm{m} / \mathrm{s})$ & $9.09 \pm 2.52 *$ & $7.94 \pm 3.39 *$ & 3.425 & 0.001 \\
\hline E/A ratio & $0.85 \pm 0.18$ & $0.89 \pm 0.17 *$ & -2.020 & 0.044 \\
\hline $\mathrm{E} / \mathrm{Em}$ & $6.87 \pm 1.54 *$ & $8.40 \pm 2.59$ & -6.352 & $<0.001$ \\
\hline
\end{tabular}

Notes: Data presented as mean \pm standard. E and $A$ early and late mitral valvular blood flow velocity peak, Em and Am mitral annular early and late peak velocity. ${ }^{*} p$ value $\leq$ 0.05 versus Before participating in this trial.

Abbreviations: IVSd, end-diastolic inter-ventricular septum thickness; LVPWTd, left ventricular posterior wall thickness; LVDd, left ventricular end-diastolic diameter; LVDs, left ventricular end-systolic diameter; LVEF, left ventricular ejection fraction; LVMI, left ventricular mass index. 
Table 3 Changes of Blood Pressure and Strain Parameters in the Two Groups of Patients After Participating in This Trial for One Year

\begin{tabular}{|c|c|c|c|c|}
\hline Parameter & $\begin{array}{c}\text { Intensive Group } \\
n=159\end{array}$ & $\begin{array}{c}\text { Standard Group } \\
n=154\end{array}$ & $\boldsymbol{t}$ & $P$ value \\
\hline \multicolumn{5}{|l|}{ Before participating in this trial } \\
\hline $\mathrm{SBP}(\mathrm{mm} \mathrm{Hg})$ & $143.70 \pm 20.65$ & $143.43 \pm 14.42$ & 0.134 & 0.893 \\
\hline $\mathrm{DBP}(\mathrm{mm} \mathrm{Hg})$ & $80.83 \pm 9.92$ & $82.43 \pm 9.65$ & -1.445 & 0.150 \\
\hline GLS-end(\%) & $-22.58 \pm 3.11$ & $-23.04 \pm 3.07$ & 1.310 & 0.191 \\
\hline GLS-mid(\%) & $-20.06 \pm 3.27$ & $-19.80 \pm 3.45$ & -0.686 & 0.493 \\
\hline GLS-epi(\%) & $-17.69 \pm 3.09$ & $-17.80 \pm 2.82$ & 0.316 & 0.752 \\
\hline \multicolumn{5}{|l|}{$\begin{array}{l}\text { After participating in this trial for } \\
\text { I year }\end{array}$} \\
\hline $\mathrm{SBP}(\mathrm{mm} \mathrm{Hg})$ & $|26.87 \pm| 4.87 *$ & $|35.52 \pm| 6.3 \mid *$ & -4.904 & $<0.001$ \\
\hline $\mathrm{DBP}(\mathrm{mm} \mathrm{Hg})$ & $77.95 \pm 8.57^{*}$ & $79.33 \pm 9.97^{*}$ & $-1.31 \mid$ & 0.191 \\
\hline GLS-end(\%) & $-23.78 \pm 3.10^{*}$ & $-23.62 \pm 3.14$ & -0.453 & 0.651 \\
\hline GLS-mid(\%) & $-20.75 \pm 3.28$ & $-20.32 \pm 3.46$ & -1.144 & 0.254 \\
\hline GLS-epi(\%) & $-18.28 \pm 3.08$ & $-|8.36 \pm 2.8|$ & 0.233 & 0.816 \\
\hline \multicolumn{5}{|l|}{ GLS improvement value after I year } \\
\hline$\Delta$ GLS-end(\%) & $1.20 \pm 0.23$ & $0.67 \pm 0.48$ & 12.415 & $<0.001$ \\
\hline$\Delta \mathrm{GLS}-\operatorname{mid}(\%)$ & $0.70 \pm 0.21$ & $0.52 \pm 0.17$ & 8.229 & $<0.001$ \\
\hline$\Delta$ GLS-epid(\%) & $0.59 \pm 0.16$ & $0.56 \pm 0.14$ & 1.665 & 0.097 \\
\hline
\end{tabular}

Notes: Data presented as mean \pm standard deviation. *p value $\leq 0.05$ versus Before participating in this trial.

Abbreviations: SBP, systolic blood pressure; DBP, diastolic blood pressure; GLS-end, GLS-mid and GLS-epi, global longitudinal strain of left ventricular endocardial layer, middle layer and epicardial layer; $\Delta \mathrm{GLS}$-end $\Delta \mathrm{GLS}$-mid and $\Delta \mathrm{GLS}$-epid, improvement value after one year of global longitudinal strain of left ventricle layers.

the intensive group were significantly higher than those in the standard group (all $\mathrm{P}<0.05$ ). There was no significant difference in the distribution of a calcium antagonist between the two groups.

\section{Changes in the Two Groups of Patients at I Year}

We found that SBP was significantly reduced at 1 year $(\mathrm{P}<0.05)$. Additionally, SBP in the intensive group was

Table 4 Antihypertensive Medication After Joining This Trial of the Two Groups

\begin{tabular}{|c|c|c|c|c|}
\hline Parameter & $\begin{array}{l}\text { Intensive Group } \\
\qquad n=159\end{array}$ & $\begin{array}{c}\text { Standard Group } \\
n=154\end{array}$ & $\chi^{2}$ & $P$ value \\
\hline $\begin{array}{l}\text { Angiotensin II type I receptor antagonists (Olmesartan Medoxomil } \\
\text { Tablets) } \\
\text { Omg } \\
5 \mathrm{mg} \\
\text { IOmg } \\
20 \mathrm{mg}\end{array}$ & $\begin{array}{l}62(39.0 \%) \\
2(1.3 \%) \\
4 I(25.7 \%) \\
54(34.0 \%)\end{array}$ & $\begin{array}{l}99(64.3 \%) \\
2(1.3 \%) \\
25(16.2 \%) \\
28(18.2 \%)\end{array}$ & 20.805 & 0.000 \\
\hline $\begin{array}{l}\text { Calcium antagonists (Amlodipine Besylate Tablets) } \\
0 \mathrm{mg} \\
\mathrm{I}-2 \mathrm{mg} \\
2.5-5 \mathrm{mg} \\
5.5-10 \mathrm{mg}\end{array}$ & $\begin{array}{l}16(10.0 \%) \\
2(1.3 \%) \\
130(81.8 \%) \\
11(6.9 \%)\end{array}$ & $\begin{array}{c}10(6.5 \%) \\
6(3.9 \%) \\
134(87.0 \%) \\
4(2.6 \%)\end{array}$ & 6.868 & 0.082 \\
\hline $\begin{array}{l}\text { Diuretics (Hydrochlorothiazide) } \\
\text { Omg } \\
10 \mathrm{mg} \\
12.5 \mathrm{mg} \\
25 \mathrm{mg}\end{array}$ & $\begin{array}{c}135(84.9 \%) \\
2(1.3 \%) \\
6(3.8 \%) \\
16(10.0 \%)\end{array}$ & 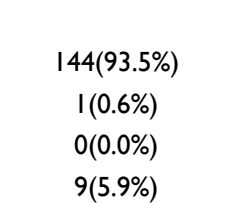 & 10.855 & 0.017 \\
\hline
\end{tabular}

Note: Data presented as n (\%). 

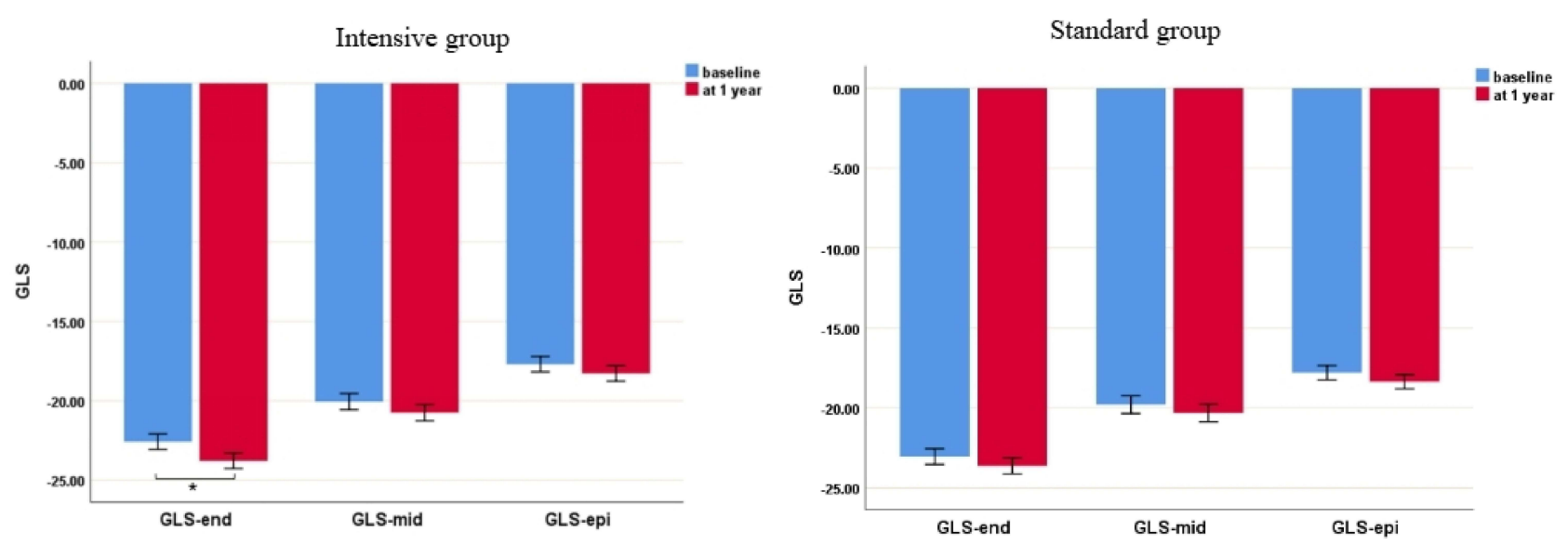

Figure 3 Changes of strain parameters in the two groups of patients after participating in this trial for one year. ${ }^{*} p$ value $\leq 0.05$ versus before participating in this trial. Abbreviations: GLS, global longitudinal strain; GLS-end, GLS-mid and GLS-epi, global longitudinal strain of left ventricular endocardial layer, middle layer and epicardial layer.

significantly lower than that in the standard group at 1 year $(\mathrm{P}<0.05)$ (Table 3).

The left ventricular structure and function parameters are shown in Table 2. After 1 year of starting the trial, in the two groups of patients, the left ventricular end-systolic diameters (LVDs) increased, LV ejection fraction decreased slightly, and Em increased. Additionally, Em of the Intensive group was higher than that of the Standard group, and E/Em was lower than that of the Standard group.

At 1 year, GLS-end in the intensive group was slightly improved compared with that before the trial $(\mathrm{P}<0.05)$
(Table 3, Figure 3). Furthermore, the improvement of the strain value ( $\Delta \mathrm{GLS}$-end and $\Delta \mathrm{GLS}$-mid) in the intensive group was higher than that in the standard group (both $\mathrm{P}<0.05$ ) (Table 3, Figure 4). There was no change in other strain parameters in older hypertensive patients within the groups after 1 year.

\section{Factors Affecting the $\Delta \mathrm{GLS}$}

To examine the factors affecting the improvement of the strain value, the medication regimen and current blood pressure were tested using multivariate backward stepwise

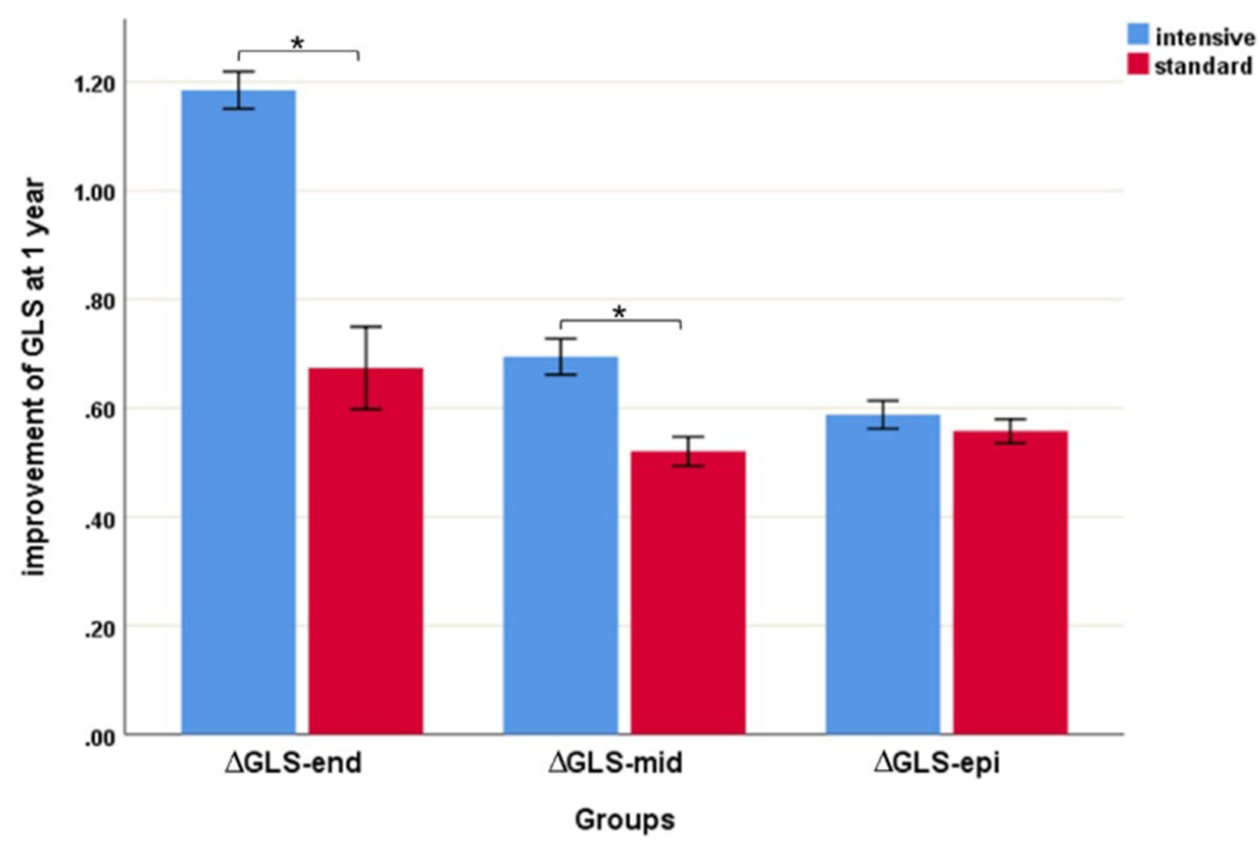

Figure 4 Comparison of GLS improvement in intensive and standard groups after one year. * $p$ value $\leq 0.05$ versus standard group. Abbreviations: GLS, global longitudinal strain; $\Delta \mathrm{GLS}$-end $\Delta \mathrm{GLS}$-mid and $\Delta \mathrm{GLS}$-epid, improvement value after one year of global longitudinal strain of left-ventricle layers. 


\begin{tabular}{|c|c|c|c|c|c|c|}
\hline \multirow{3}{*}{ 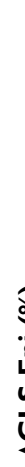 } & 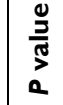 & $\begin{array}{l}\bar{\alpha} \\
\stackrel{0}{0}\end{array}$ & $\begin{array}{l}\tilde{\sigma} \\
\text { ơ } \\
0\end{array}$ & $\begin{array}{l}\text { :ơ } \\
0 \\
0\end{array}$ & $\begin{array}{l}\stackrel{2}{R} \\
0 \\
0\end{array}$ & $\begin{array}{l}\mathbb{N} \\
0 \\
0 \\
0\end{array}$ \\
\hline & $\begin{array}{l}\bar{v} \\
\text { 우 }\end{array}$ & $\begin{array}{l}\frac{n}{0} \\
\bar{i} \\
\frac{i}{\overline{0}} \\
\dot{i}\end{array}$ & 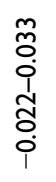 & 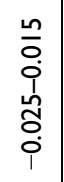 & $\begin{array}{l}\overline{8} \\
0 \\
\frac{1}{8} \\
\overline{0} \\
i\end{array}$ & $\begin{array}{l}\tilde{8} \\
0 \\
1 \\
\\
0 \\
0 \\
i\end{array}$ \\
\hline & $\simeq$ & ઠ̊. & ঃั & $\begin{array}{l}2 \\
0 \\
0 \\
0\end{array}$ & $\stackrel{\circ}{\circ}$ & $\stackrel{\circ}{\circ}$ \\
\hline & 总 & 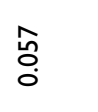 & 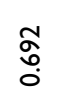 & 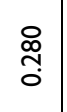 & 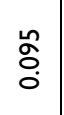 & $\begin{array}{c}m \\
\stackrel{m}{0} \\
0\end{array}$ \\
\hline & $\begin{array}{l}\bar{U} \\
\grave{\circ} \\
\text { 으 }\end{array}$ & 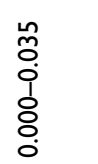 & $\begin{array}{l}\text { ợ } \\
0 \\
0 \\
0 \\
0 \\
0 \\
0 \\
0\end{array}$ & 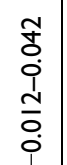 & $\begin{array}{l}8 \\
0 \\
0 \\
0 \\
0 \\
0 \\
0\end{array}$ & $\begin{array}{l}4 \\
8 \\
0 \\
1 \\
8 \\
0 \\
0\end{array}$ \\
\hline & 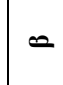 & oㅗㅇ & $\stackrel{\circ}{0}$ & $\frac{n}{0}$ & $\begin{array}{l}\overline{8} \\
\bar{i}\end{array}$ & $\overline{8}$ \\
\hline \multirow{3}{*}{ 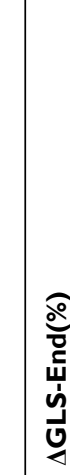 } & 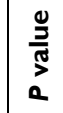 & ষ্ণ & 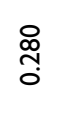 & 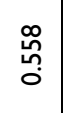 & $\hat{\circ}$ & $\frac{\hat{A}}{0}$ \\
\hline & $\begin{array}{l}\bar{v} \\
\text { วั } \\
\text { นू }\end{array}$ & \begin{tabular}{l} 
Ò \\
\hdashline 0 \\
0 \\
0 \\
0 \\
0 \\
0
\end{tabular} & 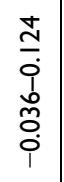 & $\mid \begin{array}{l}0 \\
0 \\
0 \\
0 \\
1 \\
0 \\
0 \\
0 \\
1\end{array}$ & $\begin{array}{l}\overline{8} \\
i \\
0 \\
0 \\
0 \\
0 \\
i\end{array}$ & $\begin{array}{l}\tau \\
\delta \\
0 \\
1 \\
\delta \\
0 \\
0 \\
i\end{array}$ \\
\hline & $=$ & $\stackrel{m}{0}$ & \begin{tabular}{l}
$\forall$ \\
\multirow{2}{0}{} \\
0
\end{tabular} & $\bar{a}$ & $\begin{array}{l} \pm \\
0 \\
0 \\
i\end{array}$ & $\begin{array}{l} \pm \\
0 \\
0 \\
0\end{array}$ \\
\hline & & 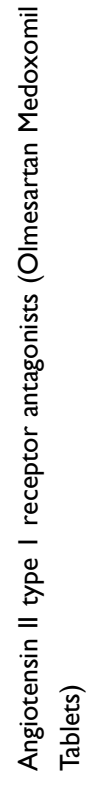 & 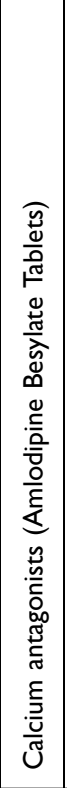 & 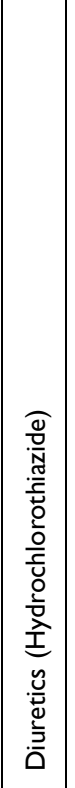 & 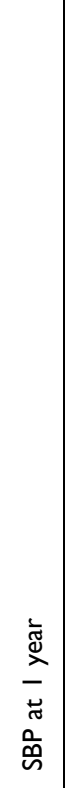 & 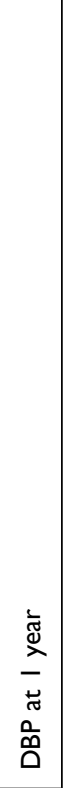 \\
\hline
\end{tabular}

linear regression analysis (Table 5). SBP at 1 year and an angiotensin II type 1 receptor antagonist were independent factors that affected $\Delta$ GLS-end $(\beta=-0.004, \quad \mathrm{P}=0.007$; $\beta=0.083, \mathrm{P}<0.001$, respectively).

\section{Intra-Observer and Inter-Observer Variability}

Table 6 shows intra- and inter-observer variability for GLS-end, GLS-mid and GLS-epi. The ICCs for intraand inter-observer variability were $0.970-0.982$ and $0.875-0.958$, respectively, which suggested that GLS in each layer of the LV was consistent.

\section{Discussion}

The main findings of our study were as follows: (1) One year after enrolling in the single-center SPRINT, GLS-end in the intensive group was slightly improved compared with that before the trial. (2) The degree of improvement in myocardial strain ( $\Delta$ GLS-end and $\Delta$ GLS-mid) in the intensive group was higher than that in the standard group. (3) SBP at 1 year and the dosage of olmesartan were independently associated with $\Delta$ GLS-end.

After enrolling in the study, the patient's adherence to antihypertensive drugs increased. Therefore, blood pressure control in older hypertensive patients in this study was more stable compared with previously. Doctors adjusted the treatment plan on the basis of the patient's daily change in blood pressure, which was generally controlled at the target level (ie, intensive group: target SBP of $110-130 \mathrm{mmHg}$ and standard group: target SBP of 130-150 mmHg). Generally, after actively controlled antihypertensive treatment can bring about improvement in LV diastolic function. ${ }^{18}$ After one year, the Em value of mitral valve in the hypertensive

Table 6 Intar-Observer and Inter-Observer Variability of Left Ventricular Strain Parameters $(n=20)$

\begin{tabular}{|c|c|c|c|c|}
\hline Parameter & $\begin{array}{c}\text { Intra- } \\
\text { Observer } \\
(n=10) \text { ICC }\end{array}$ & $95 \% \mathrm{Cl}$ & $\begin{array}{c}\text { Inter- } \\
\text { Observer } \\
(n=10) \text { ICC }\end{array}$ & $95 \% \mathrm{Cl}$ \\
\hline $\begin{array}{l}\text { GLS-end } \\
(\%)\end{array}$ & 0.981 & $0.950-0.998$ & 0.904 & $0.669-0.979$ \\
\hline $\begin{array}{l}\text { GLS-mid } \\
(\%)\end{array}$ & 0.982 & $0.957-0.998$ & 0.875 & $0.672-0.963$ \\
\hline $\begin{array}{l}\text { GLS-epi } \\
(\%)\end{array}$ & 0.970 & $0.914-0.995$ & 0.958 & $0.903-0.990$ \\
\hline
\end{tabular}

Abbreviations: ICC, intra-class coefficient; Cl, confidence interval; GLS-end, GLSmid and GLS-epi, global longitudinal strain of left ventricular endocardial layer, middle layer and epicardial layer. 
patients in this trial increased, and the diastolic function parameter E/Em of the intensive group decreased and was lower than that of the standard group, indicating that the diastolic function was improved. In patients with hypertension without complications, the LVEF is generally normal at rest, ${ }^{19}$ the LVEF of older hypertensive patients in this study was in the normal range and slightly increased after 1 year.

A previous study showed that changes in LV strain were accompanied by myocardial fibrosis and hypertrophy of cardiomyocytes. ${ }^{20}$ In our study, the intensive group had lower SBP compared with the standard group. This finding suggested that patients in the intensive group had lower cardiac afterload, less cardiac work, less myocardial oxygen consumption, milder myocardial fibrosis, less hypertrophy of cardiomyocytes, and better myocardial compliance, which resulted in higher GLS. ${ }^{21}$ Patients with higher GLS may have a lower risk of incident heart failure, acute myocardial infarction, or cardiovascular death. ${ }^{22}$ Patients in the intensive group may have a lower risk of cardiovascular events in the future, ${ }^{22}$ as suggested by the results of a large number of SPRINT studies in recent years. ${ }^{8}$ However, follow-up of our patients should be conducted in the future.

It is known that elevated blood pressure and increased LV mass in hypertensives lead to ischemia ${ }^{23}$ and early fibrosis of the subendocardial fibers. ${ }^{24,25}$ As longitudinal strain of LV is mainly attributed to subendocardial fibers. ${ }^{20}$ Investigators have found that the endocardium is vulnerable to the effect of LV filling pressure, and its function is easily impaired in patients with hypertension. ${ }^{26}$ Conversely, when the afterload of the LV is reduced, the endocardial myocardial function should be restored earlier. $^{27,28}$ Our study showed that the degree of improvement of subendocardial myocardial strain in the intensive group was higher than that in the standard group.

Olmesartan is a selective angiotensin II type 1 receptor antagonist. Angiotensin receptor blockers have been effectively used in hypertension, cardiac remodeling, and heart failure. ${ }^{29}$ And it may be used to improve the vascular smooth muscle relaxation and endothelial function, ${ }^{30}$ reverse smooth muscle cell hypertrophy ${ }^{31}$ and increase the ratio of elastin to collagen, ${ }^{32}$ resulting in a reduction in coronary artery wall thickness and improved myocardial blood supply. Additionally, angiotensin receptor blocker act on human cardiac fibroblasts directly to prevent maladaptive cardiac fibrosis and dysfunction during pressure overload-induced hypertrophy. ${ }^{33}$ Therefore, olmesartan can reverse LV remodeling and LV hypertrophy, and improve LV myocardial function. ${ }^{34,35}$ In our study, the dose of olmesartan in the intensive group was higher than that in the standard group. This resulted in a lower SBP at 1 year in the intensive group than in the standard group. And, the degree of improvement of myocardial strain in the intensive group was higher than that in the standard group. After multiple linear regression analysis, we found that the SBP at 1 year and the dosage of olmesartan were independent predictors of the degree of recovery of myocardial strain.

\section{Limitations}

The main limitation of this study is that it was a singlecenter study with a limited population. Additionally, there were no obvious complications in this group of older patients with hypertension. Therefore, the sample was biased. Future research should address this issue. Moreover, the follow-up time for this study was short. A longer follow-up is required to better understand the effect of blood pressure targets on myocardial function in older patients with hypertension.

\section{Conclusion}

In this actively controlled study for older hypertensive patients, a lower systolic blood pressure target (110-130 $\mathrm{mmHg}$ ) was beneficial for myocardial mechanics in the short term. This lowering of blood pressure has a certain protective effect on LV myocardial function.

\section{Data Sharing Statement}

All data generated or analyzed during this study are included in this article.

\section{Author Contributions}

All authors made a significant contribution to the work reported, whether that is in the conception, study design, execution, acquisition of data, analysis and interpretation, or in all these areas; took part in drafting, revising or critically reviewing the article; gave final approval of the version to be published; have agreed on the journal to which the article has been submitted; and agree to be accountable for all aspects of the work.

\section{Funding}

This work was supported by Shanxi Province Key Research and Development Plan (Grant Numbers: 201903D321199). 


\section{Disclosure}

The authors report no relationships that could be construed as a conflict of interest.

\section{References}

1. Rahimi K, Emdin CA, MacMahon S. The epidemiology of blood pressure and its worldwide management. Circ Res. 2015;116 (6):925-936. doi:10.1161/CIRCRESAHA.116.304723

2. Ettehad D, Emdin CA, Kiran A, et al. Blood pressure lowering for prevention of cardiovascular disease and death: a systematic review and meta-analysis. Lancet. 2016;387(10022):957-967. doi:10.1016/ S0140-6736(15)01225-8

3. Chobanian AV, Bakris GL, Black HR, et al.; National Heart, Lung, and Blood Institute Joint National Committee on Prevention, Detection, Evaluation, and Treatment of High Blood Pressure; National High Blood Pressure Education Program Coordinating Committee. The seventh report of the joint national committee on prevention, detection, evaluation, and treatment of high blood pressure: the JNC 7 report. JAMA. 2003;289(19):2560-2572. doi:10.1001/jama.289.19.2560

4. Neal B, MacMahon S, Chapman N, Blood Pressure Lowering Treatment Trialists' Collaboration. Effects of ACE inhibitors, calcium antagonists, and other blood-pressure-lowering drugs: results of prospectively designed overviews of randomised trials. Lancet. 2000;356(9246):1955-1964. doi:10.1016/s0140-6736(00)03307-9

5. Williams B, Mancia G, Spiering W, et al. 2018 ESC/ESH guidelines for the management of arterial hypertension. Eur Heart J. 2018;39 (33):3021-3104. doi:10.1093/eurheartj/ehy339

6. Reboussin DM, Allen NB, Griswold ME, et al. Systematic review for the 2017 ACC/AHA/AAPA/ABC/ACPM/AGS/APhA/ASH/ASPC/ NMA/PCNA guideline for the prevention, detection, evaluation, and management of high blood pressure in adults: a report of the American College of Cardiology/American Heart Association task force on clinical practice guidelines. J Am Coll Cardiol. 2018;71 (19):2176-2198. doi:10.1016/j.jacc.2017.11.004

7. Hua Q, Fan L, Jing L. Joint committee for guideline revision. 2019 Chinese guideline for the management of hypertension in the elderly. J Geriatr Cardiol. 2019;16(2):67-99. doi:10.11909/j.issn.16715411.2019.02.001

8. Wright JT Jr, Williamson JD, Whelton PK, et al; SPRINT Research Group. A randomized trial of intensive versus standard blood-pressure control. $N$ Engl J Med. 2015;373(22):2103-2116. doi:10.1056/NEJMoa1511939

9. Ma S MD, Jd W MD. New guidelines and SPRINT results implications for geriatric hypertension. Circulation. 2019;140:976-978. doi:10.1161/CIRCULATIONAHA.119.037872

10. Rocco MV, Sink KM, Lovato LC, et al.; SPRINT Research Group. Effects of intensive blood pressure treatment on acute kidney injury events in the systolic blood pressure intervention trial (SPRINT). $\mathrm{Am}$ J Kidney Dis. 2018;71(3):352-361. doi:10.1053/j.ajkd.2017.08.021

11. Jones CD, Loehr L, Franceschini N, et al. Orthostatic hypotension as a risk factor for incident heart failure: the atherosclerosis risk in communities study. Hypertension. 2012;59(5):913-918. doi:10.1161/HYPERTENSIONAHA.111.188151

12. Schmieder RE, Messerli FH, Sturgill D, Garavaglia GE, Nunez BD. Cardiac performance after reduction of myocardial hypertrophy. $\mathrm{Am}$ J Med. 1989;87:22-27. doi:10.1016/S0002-9343(89)80478-4

13. Schulman SP, Weiss JL, Becker LC, et al. The effects of antihypertensive therapy on LV mass in elderly patients. $N$ Engl $J$ Med. 1990;322:1350-1356. doi:10.1056/NEJM199005103221904

14. D'Amato A, Mancusi C, Losi MA, et al. Target organ damage and target systolic blood pressure in clinical practice: the Campania salute network. Am J Hypertens. 2018;31(6):658-664. doi:10.1093/ajh/ hpy007
15. Smiseth OA, Torp H, Opdahl A, Haugaa KH, Urheim S. Myocardial strain imaging: how useful is it in clinical decision making? Eur Heart J. 2016;37(15):1196-1207. doi:10.1093/eurheartj/ehv529

16. Marwick TH, Gillebert TC, Aurigemma G, et al. Recommendations on the use of echocardiography in adult hypertension: a report from the European Association of Cardiovascular Imaging (EACVI) and the American Society of Echocardiography (ASE). Eur Heart J Cardiovasc Imaging. 2015;16(6):577-605. doi:10.1093/ehjci/jev076

17. Lang RM, Bierig M, Devereux RB, et al; American Society of Echocardiography's Nomenclature and Standards Committee; Task Force on Chamber Quantification; American College of Cardiology Echocardiography Committee; American Heart Association; European Association of Echocardiography, European Society of Cardiology. Recommendations for chamber quantification. Eur $J$ Echocardiogr. 2006;7(2):79-108. doi:10.1016/j.euje.2005.12.014

18. Schulman DS, Flores AR, Tugoen J, Dianzumba S, Reichek N. Antihypertensive treatment in hypertensive patients with normal left ventricular mass is associated with left ventricular remodeling and improved diastolic function. Am $J$ Cardiol. 1996;78:56-60. doi:10.1016/S0002-9149(96)00227-5

19. Fouad FM. Cardiac function and dysfunction in hypertension. Cleve Clin J Med. 1994;61(5):351-355. doi:10.3949/ccjm.61.5.351

20. Ishizu T, Seo Y, Kameda Y, et al. Left ventricular strain and transmural distribution of structural remodeling in hypertensive heart disease. Hypertension. 2014;63(3):500-506. doi:10.1161/ HYPERTENSIONAHA.113.02149

21. Murai D, Yamada S, Hayashi T, et al. Relationships of left ventricular strain and strain rate to wall stress and their afterload dependency. Heart Vessels. 2017;32(5):574-583. doi:10.1007/s00380-016-0900-4

22. Biering-Sorensen T, Biering-Sorensen SR, Olsen FJ, et al. Global longitudinal strain by echocardiography predicts long-term risk of cardiovascular morbidity and mortality in a low-risk general population: the Copenhagen city heart study. Circ Cardiovasc Imaging. 2017;10:e05521. doi:10.1161/CIRCIMAGING.116.005521

23. Yano M, Kohno M, Kobayashi S, et al. Influence of timing and magnitude of arterial wave reflection on left ventricular relaxation. Am J Physiol Heart Circ Physiol. 2001;280(4):H1846-H1852. doi:10.1152/ajpheart.2001.280.4.H1846

24. Kang SJ, Lim HS, Choi BJ, et al. Longitudinal strain and torsion assessed by two-dimensional speckle tracking correlate with the serum level of tissue inhibitor of matrix metalloproteinase-1, a marker of myocardial fibrosis, in patients with hypertension. $J$ Am Soc Echocardiogr. 2008;21(8):907-911. doi:10.1016/j.echo.2008.01.015

25. Lang RM, Badano LP, Mor-Avi V, et al. Recommendations for cardiac chamber quantification by echocardiography in adults: an update from the American Society of Echocardiography and the European Association of Cardiovascular Imaging. $J \mathrm{Am} S \mathrm{Soc}$ Echocardiogr. 2015;28(1):1-39.e14. doi:10.1016/j.echo.2014.10.003

26. Nakamura N, Hirata K, Imanishi T, et al. Electrocardiographic strain and endomyocardial radial strain in hypertensive patients. Int J Cardiol. 2011;150(3):319-324. doi:10.1016/j.ijcard.2010.04.049

27. Duan F, Xie M, Wang X, et al. Preliminary clinical study of left ventricular myocardial strain in patients with non-ischemic dilated cardiomyopathy by three-dimensional speckle tracking imaging. Cardiovasc Ultrasound. 2012;10:8. doi:10.1186/1476-7120-10-8

28. Barbosa MM, Manoel O, Rocha C, et al. Early detection of left ventricular contractility abnormalities by two-dimensional speckle tracking strain in chagas'disease. Echocardiography. 2014;31 (5):623-630. doi:10.1111/echo.12426

29. Michel MC, Foster C, Brunner HR, Liu L. A systematic comparison of the properties of clinically used angiotensin II type 1 receptor antagonists. Pharmacol Rev. 2013;65(2):809-848. doi:10.1124/pr.112.007278

30. Raptis AE, Markakis KP, Mazioti MC, et al. Effect of aliskiren on circulating endothelial progenitor cells and vascular function in patients with type 2 diabetes and essential hypertension. $\mathrm{Am}$ J Hypertens. 2015;28(1):22-29. doi:10.1093/ajh/hpu119 
31. Levy BI, Michel JB, Salzmann JL, Devissaguet M, Safar ME. Remodeling of heart and arteries by chronic converting enzyme inhibition in spontaneously hypertensive rats. Am J Hypertens. 1991;4(3 Pt 2):240S-245S. doi:10.1093/ajh/4.3.240s

32. Ripley DP, Negrou K, Oliver JJ, et al. Aortic remodelling following the treatment and regression of hypertensive left ventricular hypertrophy: a cardiovascular magnetic resonance study. Clin Exp Hypertens. 2015;37(4):308-316. doi:10.3109/10641963.2014.960974

33. Burke RM, Lighthouse JK, Mickelsen DM, Small EM. sacubitril/valsartan decreases cardiac fibrosis in left ventricle pressure overload by restoring PKG signaling in cardiac fibroblasts. Circ Heart Fail. 2019;12 (4):e005565. doi:10.1161/CIRCHEARTFAILURE.118.005565
34. Regitz-Zagrosek V, Neuss M, Holzmeister J, Fleck E. Use of angiotensin II antagonists in human heart failure: function of the subtype 1 receptor. J Hypertens Suppl. 1995;13(1):S63-S71. doi:10.1097/ 00004872-199507001-00010

35. Ostroumova OD, Kochetkov AI. Effects of amlodipine/lisinopril fixed-dose combination on severity of left ventricular hypertrophy and parameters of myocardial stiffness in patients with hypertension. Kardiologiia. 2016;56(11):27-37. doi:10.18565/cardio.2016.11.27-37

\section{Publish your work in this journal}

The International Journal of General Medicine is an international, peer-reviewed open-access journal that focuses on general and internal medicine, pathogenesis, epidemiology, diagnosis, monitoring and treatment protocols. The journal is characterized by the rapid reporting of reviews, original research and clinical studies across all disease areas. The manuscript management system is completely online and includes a very quick and fair peer-review system, which is all easy to use. Visit http://www.dovepress.com/ testimonials.php to read real quotes from published authors.

Submit your manuscript here: https://www.dovepress.com/international-journal-of-general-medicine-journal 